\title{
Serum bilirubin levels on ICU admission are associated with ARDS development and mortality in sepsis
}

\author{
R Zhai, ${ }^{1}$ C C Sheu, ${ }^{1}$ L Su, ${ }^{1}$ M N Gong, ${ }^{2}$ P Tejera, ${ }^{1}$ F Chen, ${ }^{1}$ Z Wang, ${ }^{1}$ M P Convery, \\ B T Thompson, ${ }^{3}$ D C Christiani'
}

- An additional figure and additional tables are published online only at http://thorax.bmj. com/content/vol64/issue9

${ }^{1}$ Department of Environmental Health, Harvard School of Public Health, Boston, Massachusetts, USA; ${ }^{2}$ Division of Pulmonary and Critical Care Medicine,

Department of Medicine, Mount Sinai School of Medicine, New York, USA; ${ }^{3}$ Pulmonary and Critical Care Unit, Department of Medicine, Massachusetts General Hospital, Harvard Medical School, Boston, Massachusetts, USA

Correspondence to: Dr D C Christiani, Department of Environmental Health, Harvard School of Public Health, 665 Huntington Avenue, Boston, MA 02115, USA; dchris@ hsph.harvard.edu

Received 7 January 2009 Accepted 2 April 2009 Published Online First 28 May 2009
ABSTRAC

Background: Hyperbilirubinaemia is a common complication of sepsis. Elevated bilirubin may induce inflammation and apoptosis. It was hypothesised that increased serum bilirubin on Intensive Care Unit (ICU) admission contributes to sepsis-related acute respiratory distress syndrome (ARDS).

Methods: Serum bilirubin on ICU admission was measured in 1006 patients with sepsis. Serial serum bilirubin was analysed prospectively in patients with sepsis who had ARDS for a period of 28 days. The effects of clinical factors and variants of the UGT1A1 gene on serum bilirubin levels were determined. Outcomes were ARDS risk and mortality

Results: During 60-day follow-up, 326 patients with sepsis developed ARDS, of whom 144 died from ARDS. The hyperbilirubinaemia ( $\geqslant 2.0 \mathrm{mg} / \mathrm{dl}$ ) rate in patients with ARDS (22.4\%) was higher than in those without ARDS (14.1\%, $p=0.002)$. For each $1.0 \mathrm{mg} / \mathrm{dl}$ increase in admission bilirubin, ARDS risk and 28- and 60-day ARDS mortalities were increased by $7 \%(\mathrm{OR}=1.07$; $p=0.003), 20 \%(O R=1.20 ; p=0.002)$ and $18 \%$ $(O R=1.18 ; p=0.004)$, respectively. Compared with subjects with bilirubin levels $<2.0 \mathrm{mg} / \mathrm{dl}$, patients with hyperbilirubinaemia had higher risks of $\mathrm{ARDS}(\mathrm{OR}=2.12$; $p=0.0007)$ and 28-day $(O R=2.24 ; p=0.020)$ and 60 day ARDS mortalities (OR $=2.09 ; p=0.020)$. In sepsisrelated ARDS, serial bilirubin levels in non-survivors were consistently higher than in survivors $(p<0.0001)$. Clinical variables explained $29.5 \%$ of the interindividual variation in bilirubin levels, whereas genetic variants of UGT1A1 contributed $7.5 \%$.

Conclusion: In sepsis, a higher serum bilirubin level on ICU admission is associated with subsequent ARDS development and mortality.

Sepsis is the second most common cause of death in non-coronary Intensive Care Units (ICUs) and is among the top 10 causes of death for all hospitalised patients. ${ }^{1}$ Patients with sepsis are at the highest risk of developing acute respiratory distress syndrome (ARDS). ${ }^{2}{ }^{3}$ However, little is known about biomarkers predictive of ARDS development and mortality in patients with sepsis.

Bilirubin, the end-product of haem catabolism in mammals, is generally considered a lipid-soluble waste product that needs to be excreted. However, growing evidence has suggested that bilirubin at high concentrations can induce inflammation, apoptosis and oxidative stress. ${ }^{4-8}$ Hyperbilirubinaemia, or jaundice, is a well-known complication of sepsis or nonbacterial infection. ${ }^{9}$ Sepsis and bacterial infection account for $20 \%$ of jaundice cases in patients of all ages in community hospital settings. ${ }^{10}$ However, there are no data from large prospective studies on the exact incidence and prognostic relevance of hyperbilirubinaemia in adults with sepsis. ${ }^{11}$ Since most doctors view hyperbilirubinaemia as a late event in critical illness, low-grade hyperbilirubinaemia is often overlooked in patients not presenting with clinically evident jaundice. ${ }^{12}{ }^{13}$ Although hyperbilirubinaemia has been associated with overall poor outcomes in critical illness, ${ }^{12-14}$ the associations of bilirubin with ARDS risk, and factors influencing bilirubin variations in sepsis remain largely unknown.

In humans, bilirubin is mainly metabolised by uridine diphosphate glucuronosyltransferase 1A1 (UGT1A1) that contributes to bilirubin glucuronidation and thus enhances bilirubin elimination. ${ }^{15}$ The gene encoding UGT1A1 is located in chromosome 2 (2q37) and spans approximately $160 \mathrm{kbp}$. Individual genetic variations in the UGT1A1 gene, such as $-53 \sim-42\left(T_{A}\right)_{6-7}$ (UGT1A1*28, rs8175347), $-3279 T \rightarrow G(U G T 1 A 1 * 60$, rs4124874) $211 G \rightarrow A\left(U G T 1 A 1^{*} 6\right.$, rs4148323) and $-3156 G \rightarrow A$ (rs10929302) have been reported to affect UGT1A1 gene expression, enzyme activity and serum bilirubin levels. ${ }^{16-18}$ However, conflicting associations with these polymorphisms have also been reported. ${ }^{19-21}$ The apparent discrepancy suggests that a single polymorphism may not be sufficient to define the contribution of UGT1A1 variants to serum bilirubin levels. No systematic studies have addressed the association of overall genetic variation of the UGT1A1 gene with circulating bilirubin levels in critically ill patients.

The aims of the present study were to evaluate, first, whether serum bilirubin levels on ICU admission were associated with sepsis-related ARDS risk and mortality; secondly, whether clinical factors and UGT1A1 genetic variants contribute to interindividual serum bilirubin variations in sepsis; and, finally, whether UGT1A1 polymorphisms were associated with ARDS risk and mortality that were consistent with their effects on bilirubin levels.

\section{METHODS}

\section{Study subjects}

Study patients were drawn from a prospectively enrolled cohort assembled for the Molecular Epidemiology of ARDS Study. ${ }^{22}$ Consecutive admissions to the ICUs at the Massachusetts General Hospital (MGH; Boston, Massachusetts, USA) were screened for sepsis from September 
Table 1 Characteristics of 1006 patients admitted to the intensive care unit with sepsis

\begin{tabular}{|c|c|c|c|c|}
\hline Characteristics & $\begin{array}{l}\text { All patients } \\
\text { ( } \mathrm{n}=1006)\end{array}$ & $\begin{array}{l}\text { Patients who } \\
\text { developed ARDS } \\
\text { ( } \mathrm{n}=326 \text { ) }\end{array}$ & $\begin{array}{l}\text { Patients who did not } \\
\text { develop ARDS } \\
(\mathrm{n}=680)\end{array}$ & p Value \\
\hline Age, years & $62.3(17.1)$ & $59.8(17.7)$ & $63.5(16.7)$ & 0.001 \\
\hline Female & $402(40.0 \%)$ & $134(41.1 \%)$ & $268(39.4 \%)$ & 0.631 \\
\hline APACHE III score & $74.8(23.8)$ & $82.6(23.2)$ & $71.0(23.3)$ & $<0.001$ \\
\hline History of alcohol abuse & $127(12.6 \%)$ & $51(15.6 \%)$ & $76(11.2 \%)$ & 0.054 \\
\hline History of steroid use & $97(9.7 \%)$ & $33(10.1 \%)$ & $64(9.4 \%)$ & 0.733 \\
\hline Serum bilirubin (mg/dl) & $0.7(0.4-1.3)$ & $0.9(0.5-1.8)$ & $0.6(0.4-1.2)$ & $<0.0001^{*}$ \\
\hline Bilirubin $\geqslant 2.0 \mathrm{mg} / \mathrm{dl}$ & $169(16.8 \%)$ & $73(22.4 \%)$ & $96(14.1 \%)$ & 0.002 \\
\hline Serum creatinine $(\mathrm{mg} / \mathrm{dl})$ & $1.4(0.9-2.4)$ & $1.4(0.9-2.4)$ & $1.3(0.9-2.4)$ & $0.525^{*}$ \\
\hline Platelets $\left(\times 10^{9} / \mathrm{l}\right)$ & $215.8(133.5)$ & $205.0(145.2)$ & $220.9(127.3)$ & 0.005 \\
\hline Septic shock & $612(60.8 \%)$ & $234(71.8 \%)$ & $378(55.6 \%)$ & $<0.001$ \\
\hline Pneumonia & $593(59.0 \%)$ & $249(76.4 \%)$ & $344(50.6 \%)$ & $<0.001$ \\
\hline Multiple transfusion & $35(3.5 \%)$ & $11(3.4 \%)$ & $24(3.5 \%)$ & 1.00 \\
\hline End-stage renal disease & $64(6.4 \%)$ & $23(7.1 \%)$ & $41(6.0 \%)$ & 0.540 \\
\hline Trauma & $9(0.9 \%)$ & $5(1.6 \%)$ & $4(0.6 \%)$ & 0.159 \\
\hline Diabetes history & $254(25.4 \%)$ & $63(19.3 \%)$ & $191(28.2 \%)$ & 0.003 \\
\hline Liver cirrhosis/failure & $58(5.8 \%)$ & $26(8.0 \%)$ & $32(4.7 \%)$ & 0.043 \\
\hline PEEP $>5 \mathrm{~cm} \mathrm{H}{ }_{2} \mathrm{O}$ & $313(31.1 \%)$ & $176(54.0 \%)$ & $137(20.5 \%)$ & $<0.0001$ \\
\hline \multicolumn{5}{|l|}{ Microorganisms } \\
\hline Gram-positive & $432(42.9 \%)$ & $130(39.9 \%)$ & $302(44.4 \%)$ & 0.174 \\
\hline Gram-negative & $317(31.5 \%)$ & $110(33.7 \%)$ & $207(30.4 \%)$ & 0.292 \\
\hline Anaerobic & 47 (4.7\%) & $9(2.8 \%)$ & 38 (5.6\%) & 0.047 \\
\hline Fungus & $53(5.3 \%)$ & $25(7.7 \%)$ & $28(4.1 \%)$ & 0.018 \\
\hline Virus & $12(1.2 \%)$ & $2(0.6 \%)$ & $10(1.5 \%)$ & 0.241 \\
\hline Unknown & $336(33.4 \%)$ & $122(37.4 \%)$ & $214(31.5 \%)$ & 0.061 \\
\hline
\end{tabular}

Data are presented as $\mathrm{n}(\%)$, mean (SD) or median (lower quartile-upper quartile).

*Wilcoxon test.

APACHE, Acute Physiology and Chronic Health Evaluation; ARDS, acute respiratory distress syndrome; PEEP, positive endexpiratory pressure

1999 to November 2006. Sepsis was diagnosed according to the criteria of the American College of Chest Physicians/Society of Critical Care Medicine Consensus Conference. ${ }^{23}$ Exclusion criteria included age $<18$ years, diffuse alveolar haemorrhage, chronic lung diseases, directive to withhold intubation, immunosuppression except if secondary to corticosteroid, and treatment with granulocyte colony-stimulating factor (G-CSF). Alcohol abuse was defined as a history of active alcohol abuse or diagnosis for alcohol detoxification and alcoholism in the past year. ARDS was defined according to American-European Consensus Conference (AECC) criteria. $^{24}$ Organ dysfunction was measured by the ARDS Network criteria. ${ }^{25}$ Patients were followed daily for all causes of 28- and 60-day mortalities. Baseline clinical and laboratory information was collected in the first $24 \mathrm{~h}$ of ICU admission. The MGH Human Subjects Committee approved the study and informed written consent was obtained from all subjects or surrogates.

\section{Laboratory analysis}

Serum total bilirubin and other biomarkers were measured on ICU admission, using the Roche Hitachi 917 analyser with reagents from Roche Diagnostics (Indianapolis, Indiana, USA). Serial serum bilirubin levels were measured from day 1 of ARDS diagnosis until ICU discharge or death, for a period of 28 days.

Table 2 Associations of serum total bilirubin levels with sepsis-related ARDS risk and mortality: multivariate logistic regression analysis

\begin{tabular}{|c|c|c|c|c|c|c|}
\hline \multirow[b]{2}{*}{ Serum bilirubin levels } & \multicolumn{2}{|l|}{ ARDS development } & \multicolumn{2}{|c|}{ ARDS 28-day mortality } & \multicolumn{2}{|c|}{ ARDS 60-day mortality } \\
\hline & $0 R^{*}(95 \% \mathrm{CI})$ & p Value & $\mathrm{OR} \dagger(95 \% \mathrm{CI})$ & p Value & $\mathrm{OR} \dagger(95 \% \mathrm{CI})$ & p Value \\
\hline \multicolumn{7}{|l|}{ On ICU admission } \\
\hline Bilirubin & $1.07(1.03$ to 1.13$)$ & 0.003 & 1.20 (1.07 to 1.35$)$ & 0.002 & $1.18(1.05$ to 1.29$)$ & 0.004 \\
\hline Log-bilirubin & $2.17(1.46$ to 3.25$)$ & 0.0001 & $2.86(1.36$ to 6.01$)$ & 0.005 & $2.70(1.30$ to 5.61$)$ & 0.008 \\
\hline Bilirubin $\geqslant 2.0 \mathrm{mg} / \mathrm{dl}$ & 2.12 (1.37 to 3.27$)$ & 0.0007 & 2.24 (1.14 to 4.38$)$ & 0.019 & 2.09 (1.12 to 3.90$)$ & 0.020 \\
\hline \multicolumn{7}{|l|}{ On ARDS diagnosis } \\
\hline Bilirubin & - & - & 1.19 (1.06 to 1.32$)$ & 0.002 & $1.16(1.05$ to 1.28$)$ & 0.003 \\
\hline Log-bilirubin & - & - & 3.32 (1.57 to 7.06$)$ & 0.002 & 3.29 (1.57 to 6.89$)$ & 0.002 \\
\hline Bilirubin $\geqslant 2.0 \mathrm{mg} / \mathrm{dl}$ & - & - & 2.50 (1.23 to 5.08$)$ & 0.012 & 2.54 (1.33 to 4.87$)$ & 0.005 \\
\hline
\end{tabular}

${ }^{*}$ Adjustment for age, gender, clinical risks for ARDS (trauma, pneumonia, aspiration, multiple transfusions), modified APACHE (Acute Physiology and Chronic Health Evaluation) III scores, septic shock, chronic liver diseases, history of alcohol use, history of steroid use and diabetes.

$\uparrow$ Adjustment for age, gender, septic shock, chronic liver diseases, history of alcohol use, history of steroid use, diabetes, modified APACHE III scores, positive end-expiratory pressure and organ dysfunctions (respiratory, cardiovascular, renal and haematological).

ARDS, acute respiratory distress syndrome; ICU, intensive care unit. 

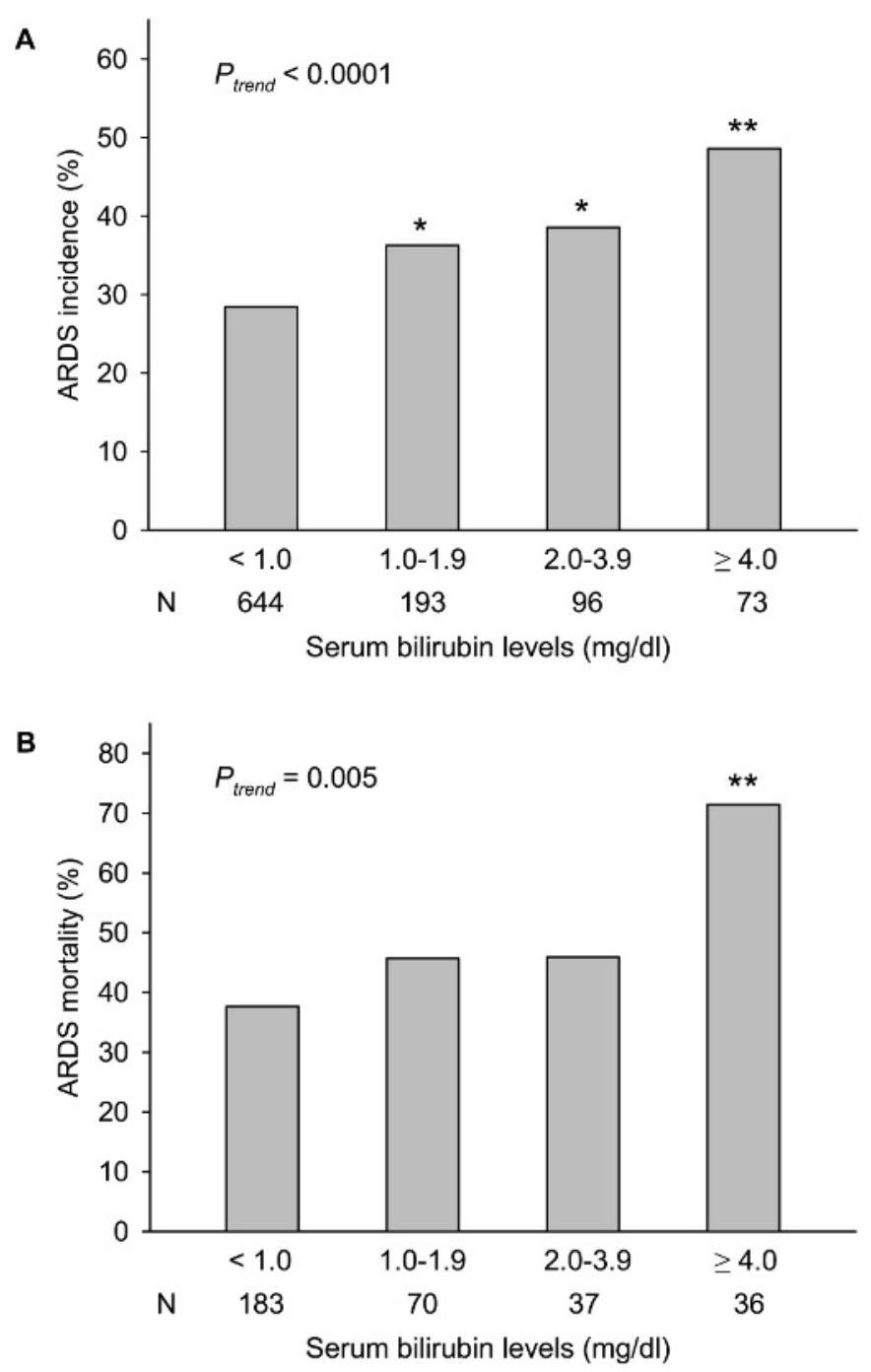

Figure 1 Distribution of incidence of acute respiratory distress syndrome (ARDS) (A) and ARDS 60-day mortality (B) by strata of serum bilirubin levels on Intensive Care Unit admission. ${ }^{*} p<0.05$ as compared with patients with bilirubin $<1.0 \mathrm{mg} / \mathrm{dl}$; ${ }^{* *} \mathrm{p}<0.01$ as compared with patients with bilirubin $<1.0 \mathrm{mg} / \mathrm{dl}$. $\mathrm{N}$, number of subjects.
Selection criteria for tagging single nucleotide polymorphisms (tSNPs) of UGT1A1 were $\mathrm{r}^{2} \geqslant 0.8$ and minor allele frequency $>5.0 \%$ across the entire UGT1A1 gene based on the database of the International HapMap Project (http://www.hapmap.org). DNA was extracted from whole blood using PureGene kits (Gentra Systems, Minneapolis, Minnesota, USA). Genotyping was determined using the Taqman assay with an ABI 7900HT sequence detector system (Applied Biosystems, Foster City, California, USA). The primer and probe sequences for each SNP are available on request. A total of $10 \%$ of samples were genotyped in duplicate for quality control and showed $100 \%$ concordance.

\section{Statistical analysis}

We compared baseline variables using the $\chi^{2}$ test, Fisher exact test, Student t test or Wilcoxon test, as appropriate. Univariate and stepwise multivariable linear regression models were used to test the associations of clinical factors and genetic variants with bilirubin levels. Since serum bilirubin levels had a skewed distribution, bilirubin levels were naturally log transformed and log bilirubin values were used in linear regression models as dependent variables. Multivariable logistic regression was used to evaluate associations of serum bilirubin levels and genetic variants of UGT1A1 with ARDS development and mortality. Association with ARDS development was adjusted for covariates including age, gender, pneumonia, aspiration, multiple transfusion, diabetes, chronic liver diseases, alcohol abuse, history of steroid use, septic shock and modified APACHE (Acute Physiology and Chronic Health Evaluation) III scores (excluding the bilirubin component). Association with ARDS mortality was adjusted for age, gender, septic shock, liver cirrhosis, history of alcohol use, history of steroid use, diabetes, modified APACHE III scores, organ dysfunctions (respiratory, cardiovascular, renal and haematological) and positive end-expiratory pressure (PEEP, defined as treatment with PEEP $>5 \mathrm{~cm} \mathrm{H}_{2} \mathrm{O}$ on ICU admission). ${ }^{26}$ Survival probability was estimated using the Kaplan-Meier log-rank test. Serial measurements were analysed by the generalised estimating equation (GEE) model.

Hardy-Weinberg equilibrium was determined using the $\chi^{2}$ test. Haplotypes were calculated using SAS macro HAPPY programs (SAS Institute, Cary, North Carolina, USA). ${ }^{22}$ Genetic covariates were analysed by additive (the wild type, heterozygotes and
Figure 2 Serial mean serum bilirubin levels after diagnosis of acute respiratory distress syndrome (ARDS) over the observation period between survivors (lower) and non-survivors (upper) in patients with sepsis-related ARDS $(n=326)(p<0.0001$, generalised estimating equation (GEE) analysis). Day 1 represents the day of ARDS diagnosis. Error bars are the SE.

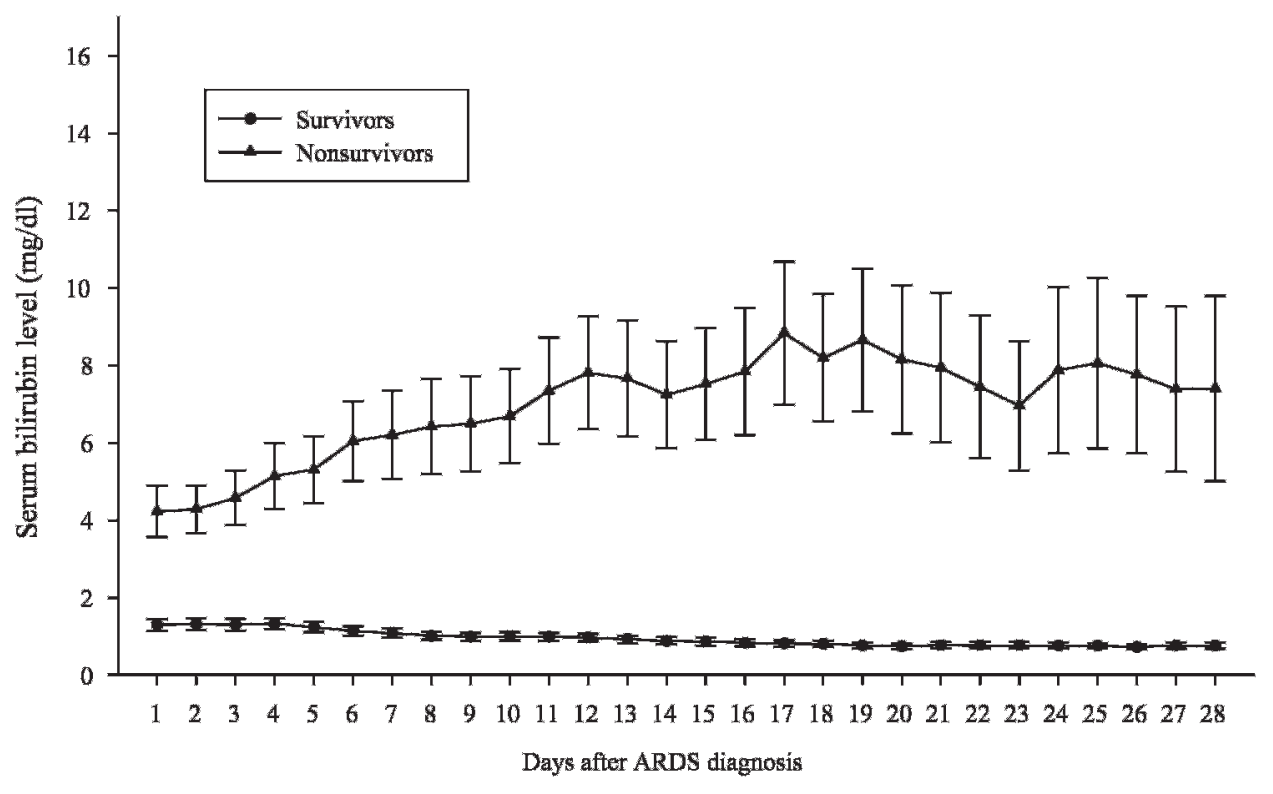




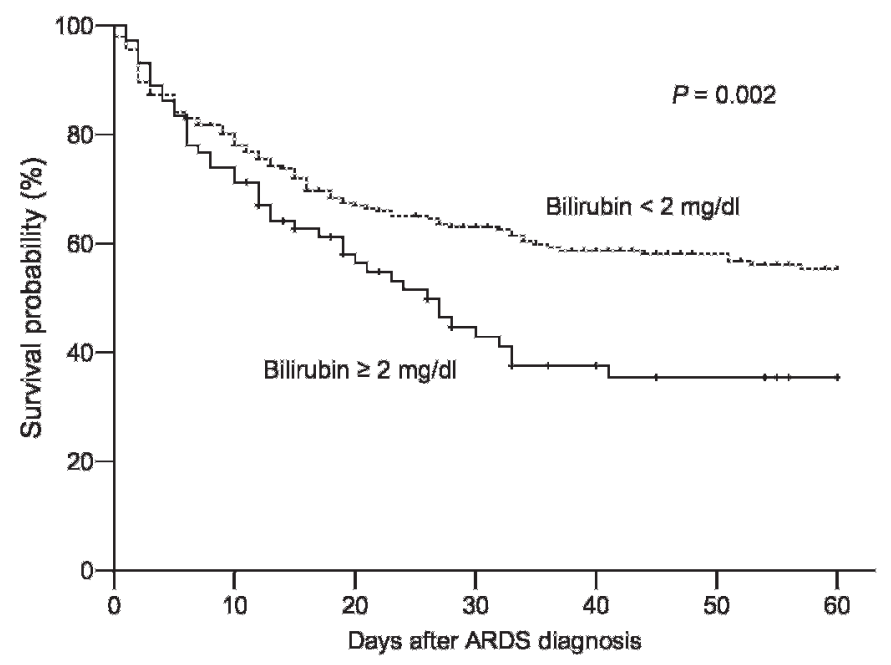

Figure 3 Estimated survival probability in patients with sepsis-related acute respiratory distress syndrome (ARDS) by admission bilirubin levels.

homozygotes were coded as 0,1 , and 2, respectively) and haplotype models. The false discovery rate (FDR) was assessed to account for multiple comparison. ${ }^{27}$ The colinearity test was performed by the SAS PROC PRINCOMP procedure.

All analyses were conducted using SAS version 9.1. A p value $<0.05$ was considered statistically significant.

\section{RESULTS}

\section{Characteristics of patients with sepsis}

Although individuals of all races were screened for this study, we restricted our analysis to Caucasians since $92 \%$ of ICU admissions during the study period at $\mathrm{MGH}$ were Caucasians. A total of 26580 consecutive ICU admissions were screened. Among them, 1931 patients meeting the criteria for sepsis and without exclusion criteria were recruited. Consent was obtained from 1224 patients with sepsis (consent rate $=63.4 \%$; Supplementary table S1). A total of 217 (18\%) patients without bilirubin records on ICU admission and one patient with genotyping failure were excluded, leaving 1006 patients for analyses. Based on the sample size of 1006 , the power of this study to detect a minimum odds ratio (OR) of 1.50 (two-sided alpha $=0.05)$ was $>80 \%$. Of the 1006 patients with sepsis, 326 (32.4\%) developed ARDS, and 144 (44.2\%) of them died within 60-day follow-up. No differences were noted between patients with and without ARDS with respect to gender, prevalence of Gram-positive and Gram-negative bacterial infection, mean creatinine levels or steroid use (table 1). Patients who developed ARDS were younger, had higher APACHE III scores, lower platelet counts and lower prevalence of diabetes. Pneumonia, chronic liver disorder and septic shock were more frequent in patients with ARDS. Serum bilirubin levels as well as the hyperbilirubinaemia rate were significantly higher in patients with sepsis who developed ARDS than those without ARDS (table 1).

\section{Associations of serum total bilirubin levels with ARDS development and mortality}

Among 1006 patients with sepsis, 169 (16.8\%) had admission bilirubin $\geqslant 2.0 \mathrm{mg} / \mathrm{dl}$ (hyperbilirubinaemia), and the remaining 837 patients had bilirubin $<2.0 \mathrm{mg} / \mathrm{dl}$. Higher serum bilirubin levels on ICU admission were significantly associated with increased ARDS incidence $\left(p_{\text {trend }}<0.0001\right.$ ) and ARDS 60-day mortality $\left(p_{\text {trend }}=0.0002\right.$ ). Interestingly, the incidence of ARDS in patients with modest bilirubin levels ( $\geqslant 1.0$ and $<2.0 \mathrm{mg} / \mathrm{dl}$ ) was significantly higher than in those with bilirubin $<1.0 \mathrm{mg} / \mathrm{dl}$ $(p=0.037)$ (fig 1). For each $1.0 \mathrm{mg} / \mathrm{dl}$ increase in admission bilirubin, ARDS risk increased by $7 \%(\mathrm{OR}=1.07$; 95\% CI, 1.03 to $1.13 ; p=0.003)$, ARDS 28-day mortality increased by $20 \%$ $(\mathrm{OR}=1.20 ; 95 \%$ CI 1.07 to $1.35 ; \mathrm{p}=0.002)$ and ARDS 60-day mortality increased by $18 \%(\mathrm{OR}=1.18$; $95 \%$ CI 1.05 to 1.31 ; $\mathrm{p}=0.004$ ). Similarly, log bilirubin values were significantly associated with ARDS development and mortalities (table 2). Bilirubin levels on ARDS diagnosis were also associated with increased mortality of sepsis-related ARDS. In sensitivity analyses by restricting the analysis to subjects without a history of chronic liver disorder $(n=948)$ and patients who did not have ARDS on ICU admission ( $n=867)$, the associations of

Table 3 Clinical correlates of serum total bilirubin levels in 1006 patients with sepsis: linear regression analysis

\begin{tabular}{|c|c|c|c|c|}
\hline \multirow[b]{2}{*}{ Clinical variables } & \multicolumn{2}{|l|}{ Univariate analysis } & \multicolumn{2}{|c|}{ Stepwise multivariate analysis } \\
\hline & Estimates (SE) & p Value & Estimates (SE) & p Value \\
\hline Age & $-0.0023(0.0008)$ & 0.004 & - & NS \\
\hline Sex, female vs male & $-0.1314(0.0278)$ & $<0.0001$ & $-0.0920(0.0244)$ & $<0.0001$ \\
\hline Septic shock & $0.0539(0.0281)$ & 0.055 & - & NS \\
\hline Diabetes & $-0.1033(0.0315)$ & 0.001 & $-0.1118(0.0275)$ & 0.0002 \\
\hline Chronic liver disorder & $0.7569(0.0538)$ & $<0.0001$ & $0.5780(0.0543)$ & $<0.0001$ \\
\hline Pneumonia & $-0.1362(0.0276)$ & $<0.0001$ & $-0.1241(0.0248)$ & $<0.0001$ \\
\hline Aspiration & $-0.0704(0.0491)$ & 0.152 & - & NS \\
\hline Multiple transfusions & $0.3756(0.0741)$ & $<0.0001$ & - & NS \\
\hline Trauma & $-0.0512(0.1460)$ & 0.700 & - & NS \\
\hline History of alcohol abuse & $0.2591(0.0405)$ & $<0.0001$ & - & NS \\
\hline History of steroid use & $-0.0824(0.0465)$ & 0.077 & - & NS \\
\hline PEEP $>5 \mathrm{~cm} \mathrm{H}_{2} \mathrm{O}$ & $0.0579(0.0296)$ & 0.051 & - & NS \\
\hline Organ dysfunction & & & & NS \\
\hline Respiratory & $0.1000(0.0294)$ & 0.0007 & $0.0928(0.0257)$ & $<0.0001$ \\
\hline Cardiovascular & $0.0638(0.0313)$ & 0.042 & - & NS \\
\hline Renal & $0.1117(0.0289)$ & 0.0001 & $0.0619(0.0256)$ & 0.022 \\
\hline Haematological & $0.4932(0.0398)$ & $<0.0001$ & $0.3018(0.0400)$ & $<0.0001$ \\
\hline
\end{tabular}

NS, not significant; PEEP, positive end-expiratory pressure. 
Table 4 Associations of UGT1A1 tagging single nucleotide polymorphisms (SNPs) with serum total bilirubin levels in patients with sepsis: linear regression analysis

\begin{tabular}{|c|c|c|c|c|c|c|c|c|c|c|}
\hline \multirow[b]{2}{*}{ LD block } & \multirow{2}{*}{$\begin{array}{l}\text { Tagging } \\
\text { SNPs }\end{array}$} & \multicolumn{3}{|c|}{ Bilirubin levels by genotypes* } & \multicolumn{3}{|l|}{ Univariate analysis } & \multicolumn{3}{|c|}{ Stepwise multivariate analysis } \\
\hline & & 0 & 1 & 2 & Estimates (SE) & p Value & FDR p & Estimates (SE) & p Value & FDR p \\
\hline \multirow[t]{12}{*}{ Block 1} & rs3755319 & 282 & 513 & 204 & & & & & & \\
\hline & & $1.64(0.26)$ & $1.62(0.16)$ & $1.86(0.24)$ & $0.0534(0.0199)$ & 0.0073 & 0.024 & $0.0493(0.0171)$ & 0.0043 & 0.005 \\
\hline & rs887829 & 438 & 455 & 108 & & & & & & \\
\hline & & $1.60(0.19)$ & $1.60(0.18)$ & $2.26(0.37)$ & $0.0694(0.0208)$ & 0.0009 & 0.0045 & $0.0609(0.0178)$ & 0.0001 & 0.0005 \\
\hline & rs6742078 & 436 & 456 & 109 & & & & & & \\
\hline & & $1.58(0.18)$ & $1.60(0.18)$ & $2.18(0.37)$ & $0.0709(0.0207)$ & 0.0006 & 0.0045 & $0.0696(0.0178)$ & 0.0001 & 0.0005 \\
\hline & rs3771342 & 757 & 227 & 21 & & & & - & & \\
\hline & & $1.75(0.15)$ & $1.36(0.13)$ & $2.02(1.29)$ & $-0.0220(0.0282)$ & 0.436 & 0.110 & - & NS & - \\
\hline & rs17864705 & 870 & 122 & 8 & & & & - & & \\
\hline & & $1.64(0.13)$ & $1.72(0.30)$ & $4.61(3.34)$ & $0.0608(0.0375)$ & 0.105 & 0.210 & - & NS & - \\
\hline & rs1018124 & 870 & 114 & 7 & & & & - & & \\
\hline & & $1.68(0.13)$ & $1.43(0.19)$ & $4.50(3.85)$ & $0.0172(0.0390)$ & 0.660 & 0.943 & - & NS & - \\
\hline Block 2 & rs11888492 & 773 & 218 & 13 & & & & - & & \\
\hline \multirow[t]{7}{*}{ Block 3} & & $1.67(0.14)$ & $1.58(0.27)$ & $3.06(1.70)$ & $-0.0002(0.0300)$ & 0.884 & 0.982 & - & NS & - \\
\hline & rs8330 & 601 & 328 & 73 & & & & - & & \\
\hline & & $1.75(0.17)$ & $1.41(0.15)$ & $2.02(0.58)$ & $-0.0103(0.0218)$ & 0.637 & 0.943 & - & NS & - \\
\hline & rs1500482 & 620 & 335 & 49 & & & & - & & \\
\hline & & $1.71(0.17)$ & $1.45(0.15)$ & $2.55(0.84)$ & $0.0003(0.0230)$ & 0.989 & 0.989 & - & NS & - \\
\hline & rs4663972 & 610 & 341 & 55 & & & & - & & \\
\hline & & $1.76(0.17)$ & $1.35(0.14)$ & $2.62(0.77)$ & $-0.0070(0.0230)$ & 0.761 & 0.951 & - & NS & - \\
\hline
\end{tabular}

Genotype 0, 1 and 2 represent the wild type, heterozygotes and homozygotes, respectively. Missing genotyping is not included.

*Data are $\mathrm{n}$ or mean (SD).

FDR, false discovery rate; LD, linkage disequilibrium.

admission bilirubin levels with sepsis-related ARDS risk and mortality remained unchanged (data not presented).

Among patients with sepsis-related ARDS, serial daily serum bilirubin levels in non-survivors were persistently higher than those in survivors from day 1 of ARDS diagnosis to the end of the entire observation period (fig 2; GEE test, $p<0.0001$ ). Kaplan-Meier survival analysis showed that patients with hyperbilirubinaemia had lower survival rates than patients with admission bilirubin $\leqslant 2.0 \mathrm{mg} / \mathrm{dl}(\mathrm{p}=0.002)$ (fig 3 ).

\section{Clinical correlates of serum total bilirubin levels on ICU admission in patients with sepsis}

In univariate linear regression analysis, age, gender, diabetes, chronic liver disorder, pneumonia, multiple transfusion, alcohol abuse and respiratory/renal/haematological dysfunctions were significantly associated with serum bilirubin levels in patients with sepsis (table 3). In stepwise multivariable linear regression analysis, chronic liver diseases, male gender and respiratory/ renal/haematological dysfunction were positively associated with ICU admission bilirubin levels, while diabetes and pneumonia were inversely related to ICU admission bilirubin levels. Clinical correlates explained $29.5 \%$ of the interindividual variation $\left(R^{2}=0.295\right)$ in serum bilirubin levels. Colinearity test suggested that colinearity among clinical factors was weak (condition number $=7.48$ ). When analysis was restricted to patients without a history of chronic liver disorders, the direction and magnitude of associations were virtually identical to results reported above (data not presented).

\section{Associations of UGT1A1 variants with serum total bilirubin levels in patients with sepsis}

The genotyping success rates for the $10 \mathrm{tSNPs}$ ranged from $99.0 \%$ to $99.5 \%$. All tSNPs in this study population were consistent with Hardy-Weinberg equilibrium ( $p>0.05, \chi^{2}$ goodness of fit). The linkage disequilibrium (LD) pattern across the UGT1A1 locus is shown in Supplementary fig S1. Three regions of strong LD were identified: block 1 ( $\sim 8 \mathrm{kbp})$, block 2 $(<1 \mathrm{kbp})$ and block $3(\sim 2 \mathrm{kbp})$. Compared with the major alleles, minor alleles of rs3755319, rs887829 and rs6742078 in LD block 1 were significantly associated with higher serum bilirubin levels (FDR $p=0.005, p=0.0005$ and $p=0.0005$, respectively; table 4). Minor alleles at rs17864705 and rs1018124 in LD block 1 also showed a trend of association with higher levels of bilirubin, but these associations did not reach statistical significance. The tSNPs explained $7.5 \%$ of the serum bilirubin

Table 5 Associations of UGT1A1 haplotypes with serum total bilirubin levels: linear regression analysis

\begin{tabular}{|c|c|c|c|c|c|c|}
\hline \multirow[b]{2}{*}{$\begin{array}{l}\text { LD } \\
\text { block }\end{array}$} & \multirow[b]{2}{*}{ Haplotype } & \multirow{2}{*}{$\begin{array}{l}\text { Haplotype } \\
\text { frequency } \\
(\%)\end{array}$} & \multicolumn{2}{|c|}{ Univariate analysis } & \multicolumn{2}{|c|}{ Multivariate analysis* } \\
\hline & & & $\begin{array}{l}\text { Estimates } \\
\text { (SE) }\end{array}$ & p Value & $\begin{array}{l}\text { Estimates } \\
\text { (SE) }\end{array}$ & p Value \\
\hline \multirow{5}{*}{$\begin{array}{l}\text { Block } \\
1 \dagger\end{array}$} & ATTTTG & 52.8 & 1.0 & - & 1.0 & - \\
\hline & CCGTTG & 32.8 & $\begin{array}{l}0.0698 \\
(0.0215)\end{array}$ & 0.0012 & $\begin{array}{l}0.0712 \\
(0.0189)\end{array}$ & 0.0002 \\
\hline & CTTGTG & 5.7 & $\begin{array}{l}-0.0169 \\
(0.0422)\end{array}$ & 0.689 & $\begin{array}{l}-0.0089 \\
(0.0368)\end{array}$ & 0.807 \\
\hline & CTTGGA & 5.6 & $\begin{array}{l}0.0737 \\
(0.0410)\end{array}$ & 0.073 & $\begin{array}{l}0.0321 \\
(0.0356)\end{array}$ & 0.380 \\
\hline & Others & 3.1 & - & - & - & - \\
\hline \multirow{4}{*}{$\begin{array}{l}\text { Block } \\
3 \$\end{array}$} & GTT & 73.6 & 1.0 & - & 1.0 & - \\
\hline & $\operatorname{cCC}$ & 20.4 & $\begin{array}{l}-0.0153 \\
(0.0235)\end{array}$ & 0.593 & $\begin{array}{l}-0.0240 \\
(0.0206)\end{array}$ & 0.244 \\
\hline & CTT & 2.53 & $\begin{array}{l}-0.0313 \\
(0.0585)\end{array}$ & 0.581 & $\begin{array}{l}-0.0179 \\
(0.0513)\end{array}$ & 0.727 \\
\hline & Others & 3.47 & - & - & - & - \\
\hline
\end{tabular}

${ }^{*}$ Adjustment for age, gender, septic shock, chronic liver diseases, history of alcoho use, history of steroid use, diabetes, modified APACHE (Acute Physiology and Chronic Health Evaluation) III scores and organ dysfunctions (respiratory, cardiovascular, renal and haematological).

†Polymorphisms are in the order: rs3755319-rs887829-rs6742078-rs3771342rs17864705-rs1018124.

tPolymorphisms are in the order: rs8330-rs1500482-rs4663972.

LD, linkage disequilibrium. 
variation $\left(\mathrm{R}^{2}=0.075\right)$ in models that included all clinical covariates. No associations between serum bilirubin and tSNPs in LD block 2 and block 3 were observed.

In $\mathrm{LD}$ block 1 , four common haplotypes with frequencies $\geqslant 5 \%$ were reconstructed (table 5). Using the most common haplotype ATTTTG (52.8\%) as the reference, haplotype CCGTTG $(32.8 \%)$ that harboured the minor alleles of rs3755319, rs887829 and rs6742078 was significantly associated with higher bilirubin levels (adjusted $p=0.0002$; global test $\mathrm{p}<0.0001$ ). No haplotype in LD block 3 was associated with bilirubin levels.

\section{Associations of UGT1A1 variants with the development and mortality of sepsis-related ARDS}

Because UGT1A1 variants were strongly associated with higher serum bilirubin levels, and higher bilirubin levels were associated with increased ARDS development and mortality, we evaluated whether UGT1A1 tSNPs or haplotypes are associated with ARDS development or mortality. The distributions of UGT1A1 tSNP genotypes were not significantly different between patients with and without ARDS (all $p$ values $>0.05$ ). In logistic regression using the major allele homozygotes as referent genotypes, rs17864705 and rs1018124 (in LD block 1) were marginally associated with increased risk of $\mathrm{ARDS}\left(\mathrm{OR}_{\mathrm{adj}}=1.52, \mathrm{p}=0.037\right.$ for $r$ 17864705; $O R_{a d j}=1.48, p=0.058$ for rs1018124). However, these associations were no longer significant in FDR analysis (Supplementary table S3). Similarly, two haplotypes were marginally associated with increased ARDS risk in overall analysis (Supplementary table S3). No significant associations were detected between any UGT1A1 tSNPs or haplotypes and ARDS mortalities.

\section{DISCUSSION}

This study shows that a higher bilirubin on ICU admission is associated with subsequent sepsis-related ARDS development and mortality. Furthermore, our study shows that serum bilirubin levels in sepsis are mainly influenced by clinical factors and partly by genetic variants of UGT1A1.

Although sepsis is a major risk for $\mathrm{ARDS},{ }^{28}$ no prior study has described predictive biomarkers for ARDS development in patients with sepsis. Our results showed that a slight increase in bilirubin on ICU admission was associated with a marked increase of ARDS risk and mortality in patients with sepsis, suggesting that serum bilirubin is an early and sensitive biomarker of sepsis-related ARDS. The fact that adjustments for multiple covariates did not change these associations further suggested that bilirubin is an independent predictor of sepsisrelated ARDS. Previous studies on the prognostic value of bilirubin in sepsis have focused mainly on survival as the major outcome measure. ${ }^{29}{ }^{30}$ Although the ultimate outcome measure for any patient is survival, death is not the only outcome measure of ICU treatment. In critically ill patients, identification of risk biomarkers for ARDS development may help clinicians in both diagnostic evaluation and management. For instance, hyperbilirubinaemia has been reported to predispose surgical ICU patients to infection, indicating a need for a higher level of vigilance for infection in ICU patients with hyperbilirubinaemia. $^{12} 31$

Our results from a large study population confirmed the findings in previous small studies that hyperbilirubinaemia is associated with worse survival in patients with ARDS. ${ }^{29} 30$ Unlike previous reports, ARDS diagnosis in our study was determined prospectively using the widely accepted AECC definition, and therefore phenotype misclassification was minimised. The use of individual phenotype rather than critical illness from a mixture causes reduces confounding from any possible associations between the various causes and outcomes. Moreover, the outcomes of ARDS were measured for 60-day mortality; therefore, bias in survival calculation due to incomplete observation was minimised.

Although physiological levels of bilirubin are considered a potent antioxidant, ${ }^{32}$ increasing evidence has shown that bilirubin at high concentrations may be an active participant in the disease process. Bilirubin in the blood can induce cell lysis of erythrocytes. ${ }^{33}$ Elevated bilirubin can stimulate oxidative stress and decrease cell survival. ${ }^{34}{ }^{35}$ Bilirubin promotes apoptosis in cultured cells. ${ }^{5}$ In addition, bilirubin can also induce the inflammatory response, which is further increased when cells are simultaneously exposed to lipopolysaccharide.

Consistent with a previous report in critically ill patients, ${ }^{13}$ we observed that several clinical factors including pneumonia, preadmission steroid use, diabetes history and organ dysfunction (respiratory, renal and haematological) were associated with serum bilirubin levels. In contrast to previous studies using univariate analysis, we defined the contributions of clinical factors to bilirubin variations by multivariate regression models and demonstrated that clinical factors explained $29.5 \%$ of the overall variation of serum bilirubin levels in patients with sepsis. It should be pointed out that although chronic liver disease is strongly associated with increased serum bilirubin levels, hepatic dysfunction is not the only organ dysfunction associated with hyperbilirubinaemia in sepsis. Cholestasis, circulating endotoxin and haemolysis may also play important roles in the aetiology of hyperbilirubinaemia in sepsis. ${ }^{11}$ In subjects without chronic liver disorders, we found that other clinical factors were also significantly associated with serum bilirubin levels. It has been reported that elevated bilirubin was often seen in patients with sepsis and septic shock in the absence of primary liver or biliary disease. ${ }^{31}$

Despite the evidence that genetic variants of UGT1A1 were significantly associated with serum bilirubin levels, the overall contribution of these variants was small compared with clinical factors. Similar to the moderate impact of genetic variants of UGT1A1 on serum bilirubin levels, the strengths of overall associations between UGT1A1 polymorphisms with sepsisrelated ARDS risk were also modest. Considering that $<8 \%$ of the variance in bilirubin levels is explained by UGT1A1 polymorphisms, it is not surprising that we did not detect a strong association of these polymorphisms with ARDS risk or mortality, although the association of serum bilirubin level with ARDS risk and mortality is relatively strong.

The large sample size, the adjustment for multiple clinical covariates, comprehensive evaluation of common variants surrounding the UGT1A1 gene and serial measurements of bilirubin levels are the strengths of this study. Despite these assets, our study has some limitations. First, our study design did not allow us to define whether bilirubin is an effecter molecule in the pathogenesis of ARDS or merely a marker of systemic injury. Future research is needed to elucidate the potential pathogenic role of bilirubin in ARDS. Secondly, this study could not exclude the possibility of medication influence on bilirubin levels. However, a recent study including 17 drugs potentially inducing hepatotoxicity has shown that administration of medications during ICU stay did not significantly contribute to serum bilirubin levels. ${ }^{13}$ Thirdly, we were unable to exclude the possibility that our cohort might include subjects with existing Gilbert syndrome because all subjects in this study 
were critically ill patients. Whether Gilbert syndrome might be associated with sepsis-related ARDS requires further investigation. Fourthly, 217 patients with sepsis were excluded from analysis due to no ICU admission bilirubin records. Since most of these patients had bilirubin measurements $<2.0 \mathrm{mg} / \mathrm{dl}$ analysed before ICU admission, they were considered as patients with normal bilirubin levels and were not tested for bilirubin by ICU clinicians. Comparison analysis showed that patients without ICU admission bilirubin values had lower severity of illness and less organ failure than those with admission bilirubin and were more similar to those with bilirubin $<1.0 \mathrm{mg} / \mathrm{dl}$ than to those patients with bilirubin $>1.0 \mathrm{mg} / \mathrm{dl}$ (Supplementary table S2). Therefore, we assumed that patients without ICU admission bilirubin values were probably among subjects with bilirubin $<1.0 \mathrm{mg} / \mathrm{dl}$, and exclusion of these patients from our analyses did not change the results, and probably diluted the strength of associations detected in this study.

In conclusion, we show that the serum bilirubin level on ICU admission is an independent early predictor of ARDS development and mortality in patients with sepsis. Several clinical characteristics and genetic variants of UGT1A1 independently affect serum bilirubin levels. However, the relationship between UGT1A1 variants and bilirubin levels did not translate largely to an associated change in ARDS outcomes.

Acknowledgements: The authors gratefully acknowledge valuable contributions to this study from the following: Marcia Chertok, Janna Frelich, Julia Shin, Andrea Shafer, Lia Shimada, Weiling Zhang, Kelly McCoy and Thomas McCabe.

Funding: This work was supported by grants from the National Institute of Health (HL60710 and ES00002) and the Flight Attendant Medical Research Institute (FAMRI, 062459 YCSA).

\section{Competing interests: None.}

Ethics approval: The MGH Human Subjects Committee approved the study.

Provenance and peer review: Not commissioned; externally peer reviewed.

\section{REFERENCES}

1. Martin GS, Mannino DM, Eaton S, et al. The epidemiology of sepsis in the United States from 1979 through 2000. N Engl J Med 2003;348:546-54.

2. Piantadosi CA, Schwartz DA. The acute respiratory distress syndrome. Ann Intern Med 2004:141:460-70

3. Hudson LD, Milberg JA, Anardi D, et al. Clinical risks for development of the acute respiratory distress syndrome. Am J Respir Crit Care Med 1995;151:293-301.

4. Noriega G0, Tomaro ML, del Batlle AM. Bilirubin is highly effective in preventing in vivo delta-aminolevulinic acid-induced oxidative cell damage. Biochim Biophys Acta 2003;1638:173-8.

5. Fernandes A, Falcao AS, Silva RF, et al. Inflammatory signalling pathways involved in astroglial activation by unconjugated bilirubin. J Neurochem 2006;96:1667-79.

6. Rodrigues $\mathbf{C M}$, Sola S, Brito MA, et al. Bilirubin directly disrupts membrane lipid polarity and fluidity, protein order, and redox status in rat mitochondria. $J$ Hepatol 2002;36:335-41.

7. Alexandra Brito M, Silva RF, Brites D. Bilirubin toxicity to human erythrocytes: a review. Clin Chim Acta 2006;374:46-56.

8. Chopra M, Reuben JS, Sharma AC. Acute lung injury: apoptosis and signaling mechanisms. Exp Biol Med (Maywood) 2009;234:361-71.

9. Fuchs M, Sanyal AJ. Sepsis and cholestasis. Clin Liver Dis 2008;12:151-72, ix
10. Whitehead MW, Hainsworth I, Kingham JG. The causes of obvious jaundice in South West Wales: perceptions versus reality. Gut 2001;48:409-13.

11. Chand N, Sanyal AJ. Sepsis-induced cholestasis. Hepatology 2007;45:230-41.

12. Field $\mathbf{E}$, Horst HM, Rubinfeld IS, et al. Hyperbilirubinemia: a risk factor for infection in the surgical intensive care unit. Am J Surg 2008;195:304-6; discussion 306-7.

13. Brienza N, Dalfino L, Cinnella G, et al. Jaundice in critical illness: promoting factors of a concealed reality. Intensive Care Med 2006;32:267-74.

14. Harbrecht BG, Zenati MS, Doyle HR, et al. Hepatic dysfunction increases length of stay and risk of death after injury. J Trauma 2002:53:517-23.

15. Tukey RH, Strassburg CP. Human UDP-glucuronosyltransferases: metabolism, expression, and disease. Annu Rev Pharmacol Toxicol 2000;40:581-616.

16. Lampe JW, Bigler J, Horner NK, et al. UDP-glucuronosyltransferase (UGT1A1*28 and UGT1A6*2) polymorphisms in Caucasians and Asians: relationships to serum bilirubin concentrations. Pharmacogenetics 1999;9:341-9.

17. Sai K, Saeki M, Saito Y, et al. UGT1A1 haplotypes associated with reduced glucuronidation and increased serum bilirubin in irinotecan-administered Japanese patients with cancer. Clin Pharmacol Ther 2004;75:501-15.

18. Saeki M, Saito Y, Sai K, et al. A combinatorial haplotype of the UDPglucuronosyltransferase $1 \mathrm{~A} 1$ gene increases total bilirubin concentrations in Japanese volunteers. Clin Chem 2007:53:356-8.

19. Miners J0, McKinnon RA, Mackenzie PI. Genetic polymorphisms of UDPglucuronosyltransferases and their functional significance. Toxicology 2002;181182:453-6.

20. Toffoli G, Cecchin E, Corona G, et al. The role of UGT1A1*28 polymorphism in the pharmacodynamics and pharmacokinetics of irinotecan in patients with metastatic colorectal cancer. J Clin Oncol 2006:24:3061-8.

21. Peterkin VC, Bauman JN, Goosen TC, et al. Limited influence of UGT1A1*28 and no effect of UGT2B7*2 polymorphisms on UGT1A1 or UGT2B7 activities and protein expression in human liver microsomes. Br J Clin Pharmacol 2007;64:458-68.

22. Zhai R, Gong MN, Zhou W, et al. Genotypes and haplotypes of the VEGF gene are associated with higher mortality and lower VEGF plasma levels in patients with ARDS. Thorax 2007:62:718-22.

23. Bone RC, Balk RA, Cerra FB, et al. Definitions for sepsis and organ failure and guidelines for the use of innovative therapies in sepsis. The ACCP/SCCM Consensus Conference Committee. American College of Chest Physicians/Society of Critical Care Medicine. Chest 1992;101:1644-55.

24. Bernard GR, Artigas A, Brigham KL, et al. Report of the American-European consensus conference on ARDS: definitions, mechanisms, relevant outcomes and clinical trial coordination. The Consensus Committee. Intensive Care Med 1994:20:225-32

25. The Acute Respiratory Distress Syndrome Network. Ventilation with lower tidal volumes as compared with traditional tidal volumes for acute lung injury and the acute respiratory distress syndrome. N Engl J Med 2000;342:1301-8.

26. Rubenfeld GD, Herridge MS. Epidemiology and outcomes of acute lung injury. Chest 2007:131:554-62.

27. Benjamini Y, Yekutieli D. Quantitative trait loci analysis using the false discovery rate. Genetics 2005;171:783-90.

28. Fein AM, Calalang-Colucci MG. Acute lung injury and acute respiratory distress syndrome in sepsis and septic shock. Crit Care Clin 2000;16:289-317.

29. Schwartz DB, Bone RC, Balk RA, et al. Hepatic dysfunction in the adult respiratory distress syndrome. Chest 1989;95:871-5.

30. Hebert PC, Drummond AJ, Singer J, et al. A simple multiple system organ failure scoring system predicts mortality of patients who have sepsis syndrome. Chest 1993:104:230-5

31. Franson TR, LaBrecque DR, Buggy BP, et al. Serial bilirubin determinations as a prognostic marker in clinical infections. Am J Med Sci 1989;297:149-52.

32. Minetti M, Mallozzi C, Di Stasi AM, et al. Bilirubin is an effective antioxidant of peroxynitrite-mediated protein oxidation in human blood plasma. Arch Biochem Biophys 1998;352:165-74.

33. Brito MA, Silva R, Tiribelli $C$, et al. Assessment of bilirubin toxicity to erythrocytes Implication in neonatal jaundice management. Eur J Clin Invest 2000:30:239-47.

34. Cesaratto $\mathbf{L}$, Calligaris SD, Vascotto $\mathrm{C}$, et al. Bilirubin-induced cell toxicity involves PTEN activation through an APE1/Ref-1-dependent pathway. J Mol Med 2007:85:1099-112.

35. Ostrow JD, Pascolo L, Brites D, et al. Molecular basis of bilirubin-induced neurotoxicity. Trends Mol Med 2004;10:65-70. 